\title{
New Online Platform Will Allow Early Release, Alt-metrics, and Extended Datasets
}

JNeurosci will be joining its sibling eNeuro on a new online platform that will allow several innovations to its format. This has been a massive undertaking, and everything possible has been done to make sure the transition is a smooth one. This preparation is almost complete, and the new platform will go live in October.

For our authors and reviewers, the migration will be seamless since there has been no change to the submission and review process. The website will be easier to navigate with a more cohesive organization providing easier access to information. More important, the new platform will allow us to make available several new features to authors and readers of JNeurosci:

- Early Release with articles posted online soon after acceptance;

- New Alt-metrics;

- Extended datasets will allow us to publish the increasingly data-intensive articles that are pushing the boundaries of neuroscience.

One goal of scientific publishing is to allow access to peerreviewed science as quickly as possible. JNeurosci works hard to keep the review process as rapid as possible, and the time to first decision remains close to $30 \mathrm{~d}$ from submission. We have also initiated consultation sessions between editors and between editors and reviewers with the goal to provide rapid decisions on manuscripts that do not fit into the scope of JNeurosci, obtain a consensus when needed, and make third reviewer invitations as rare as possible. In line with those efforts, publishing articles online shortly after they are accepted will allow the neuroscience community access to new data as quickly as possible after the peer review process.

The new online platform, which is already in use by eNeuro, allows authors to access Alt-metrics, including mentions on social media, how often the abstract, PDF, or online version of the article have been accessed, and the number of citations. Discussion about JNeurosci articles will also be facilitated, and eLetters will be highlighted alongside the article for easy response. These new features are becoming more generally used and will help authors and readers evaluate the reach of published articles.
The most significant change will be the ability to host extended datasets linked to figures and tables in the online version of the manuscript. In 2010, JNeurosci made the decision to eliminate supplemental materials because there was a concern these were difficult to access, poorly or nonreviewed, and because there was a perception that supplemental figures encouraged reviewers to ask for many more experiments, not all of which were relevant to the main data in a manuscript. Since that time, neuroscience studies are becoming more data rich, and the inability to host large datasets has made it difficult for authors to publish several types of studies in JNeurosci.

Primary data, such as gene ontology lists, protein structures, and chemical compounds, fixed in time to the publication are critical findings in many studies. In addition to the necessity of presenting the primary findings of a study, supplemental data can be important for transparency and reproducibility. Sharing unedited Western blots, traces, and other primary data, as well as scripts used for data analysis, is important for those who wish to replicate and build on published studies.

To address these issues, JNeurosci will use the new online platform to implement inclusion of Extended Data linked to figures and tables in manuscripts. We plan to complete the process and allow submission of Extended Data by the end of this year. All Extended Data will be peer-reviewed, and the editors will determine whether it is essential to the manuscript. Note that those materials for which there are publicly accessible databases should be deposited in central repositories. The goal is to keep Extended Data focused on materials that simply cannot be included in the body of the manuscript. We are also concerned that extended data are invisible to search engines used to count citations, so all citations to published studies must also appear in the main body of the paper.

We are excited that the migration to a new online platform will allow JNeurosci to provide our authors with new ways to present their scientific findings, and to address issues related to timely dissemination of data and reproducibility. As always, if you have comments or suggestions, Please e-mail me at JN_EIC@SFN.ORG or tweet @MarinaP63.

(D)Marina Picciotto, EiC, JNeurosci

DOI:10.1523/JNEUROSCI.2960-16.2016 